\title{
Performance Comparison of Energy Efficient Dynamic Transmission and Static Transmission Power in Static Mobility Node Wireless Ad-Hoc Network
}

\author{
Siti Asilah Yah ${ }^{1, *}$, Naimah Yaakob ${ }^{1}$, Ong Bi Lynn ${ }^{1}$, Mohammad Elshaikh Elobaid ${ }^{1}$, and Wan Aida Nadia Wan Abdullah ${ }^{1}$ \\ ${ }^{1}$ ENAC, School of Computer and Communication Engineering (SCCE), University Malaysia Perlis (UniMAP) 02600, \\ Arau, Perlis, Malaysia
}

\begin{abstract}
Transmission power optimization in Wireless Ad-Hoc Network is an important thing in order to minimize the energy consumption for effective utilization of the applications like Vehicle Ad-Hoc Network (VANET) applications. If one or more nodes in the wireless Ad-hoc network have little or no energy, then data transmission will be temporarily or permanently interrupted which might create a serious havoc in the Ad-hoc network especially during vital information transferred. This will, in turn, affect the performance of the entire network. Therefore transmission power control is one of the important research topics that needs to be focused in the wireless ad-hoc network in order to ensure effective energy consumption. Recently, we proposed a Dynamic Transmission Power algorithm to maintain network connectivity by adapting node's transmission power based on the distance between the vehicles in VANET. Our research aims to design a dynamic transmission power that can minimize the rate of energy consumption. Hence, in order to develop the proposed method, prerequisite experiment need to be done. This paper investigates the energy saving efficiency of dynamic and static transmission range in static mobility node wireless ad-hoc network which is prerequisite experiments before further experiment on VANET can be carried on. The simulation results prove that dynamic transmission range gives better energy consumption compared to static transmission range, so it is worth it to carry out the subsequent experiments on VANET.
\end{abstract}

\section{Introduction}

An ad hoc network is a collection of wireless mobile nodes dynamically forming a temporary network without the use of any existing network infrastructure or centralized administration [1]. In many ad hoc networks, two hosts that want to communicate may not be necessary within the wireless transmission range of each other. However, they could communicate with each other if the other hosts who are also participates in the ad hoc network are willing to forward packets for them. An advantage of the system is robustness, flexibility and mobility.

The ad hoc networks can be classified according to their application as Mobile Ad hoc Network (MANET) which is a self-arranging infrastructure-less network of mobile devices communicated through wireless link. Vehicular Ad hoc Network (VANET) uses travelling cars as nodes in a network to create a mobile network. Wireless Sensor Network (WSN) consists of autonomous sensors to control the environmental actions [2]. The nodes in the all of this application use an ad-hoc network to communicate with each other and they did not need any based station to communicate.

Since energy sources have a limited lifetime, power availability is one of the most important constraints for the operation of the ad hoc network [3]. If one or more nodes in wireless Ad-hoc network have little or no energy, then data transmission will be interrupted temporarily or permanently which might create a serious havoc in the Ad-hoc network especially when vital information is in transmission and requires an urgent response. This will in turn affect the performance of the entire network. Moreover, nodes involved in Wireless Ad-hoc network act as host and intermediate node to forward data of the neighbouring nodes and receiving node. Therefore, a lot of energy are needed to perform the task. The nodes require to use energy efficiently in order to avoid energy wasted, hence enable the nodes to perform all the given tasks. Therefore transmission power control is one of the important research topics that needs to be focused in Ad-hoc network application in order to ensure the effectiveness of energy consumption.

Recently we proposed Dynamic Transmission Power algorithm which is an algorithm to maintain network connectivity by adapting node's transmission power based on the distance between the vehicles in one of the ad hoc applications, which is VANET. The aim of the research is to design a dynamic transmission power that can minimize the rate of energy consumption. However, prerequisite experiment need to be done before the proposed method can be proceed. So, this paper investigates the energy saving efficiency of dynamic transmission range and static transmission

\footnotetext{
* Corresponding author: ilahyah_92@yahoo.com
} 
range in static mobility node wireless ad-hoc network which is prerequisite experiments before further experiment on VANET can be carry on.

The rest of the paper is organized as follows. Explanation about the methodology of this experiment are presented in section 2. The simulation setup are explained in section 2.1. In section 3, the evaluation scenario for this experiment are discussed. Then, the selected simulation metric are presented in section 3.1. Section 4 discusses about the collected result and the final section concludes the paper.

\section{Methodology}

In this paper, OMNeT++ [4], was used to design the simulation model and perform all the experiments to evaluate and analyze the effects of using different packet sizes and packet rates on the energy consumption of wireless ad-hoc network.

\subsection{Simulation Setup}

The purpose of this phase is to prove that by using dynamic transmission range, the energy consumption can be further optimized. The significant parameters need to be determined in order to model dynamic transmission power algorithm in VANET environment. The significant parameters that have been decided to be in this experiment are shown in Table 1. The parameters are used for both dynamic and fixed transmission range.

Table 1. Parameter Setup for Fixed and Dynamic Transmission Range

\begin{tabular}{|c|c|}
\hline $\begin{array}{c}\text { Parameters } \\
\text { Names }\end{array}$ & Values \\
\hline Simulation Area & $1000 \mathrm{~m} * 1000 \mathrm{~m}$ \\
\hline Simulation Time & $500 \mathrm{~s}$ \\
\hline Routing Protocol & AODV \\
\hline Transport Protocol & UDP \\
\hline Packet Size & $1,2,3,4$ and 5 KB \\
\hline Packet Rate & $2,4,6$ and 8 Packet / sec.) \\
\hline
\end{tabular}

For transport protocol, User Datagram Protocol (UDP) has been chosen because it can be very fast, with low delay and are not affected by congestion on a connection basis. On other hand, reduces the overhead with the packet header size of just 8 bytes. UDP has comparatively fast speed and is thus used for games or applications that require fast transmission of data. UDP also transmits fixed sized datagrams and can be used for multicasting. For routing protocol, AODV protocol is used because it uses a broadcast route discovery mechanism and it is responsive to changes in network topology.

Next step is to design and verify the simulation model. For this purpose, a few simulations need to be run. The details of the design simulations are described in Figure 1 and Figure 2.

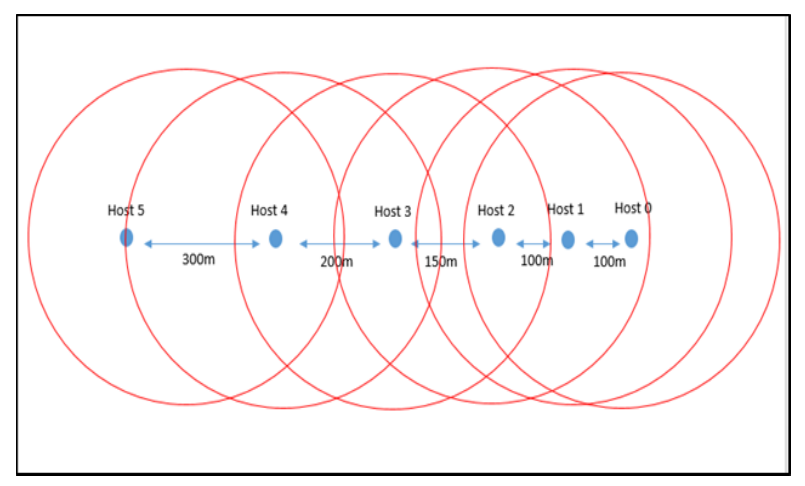

Fig.1. Fixed Transmission Range Illustration

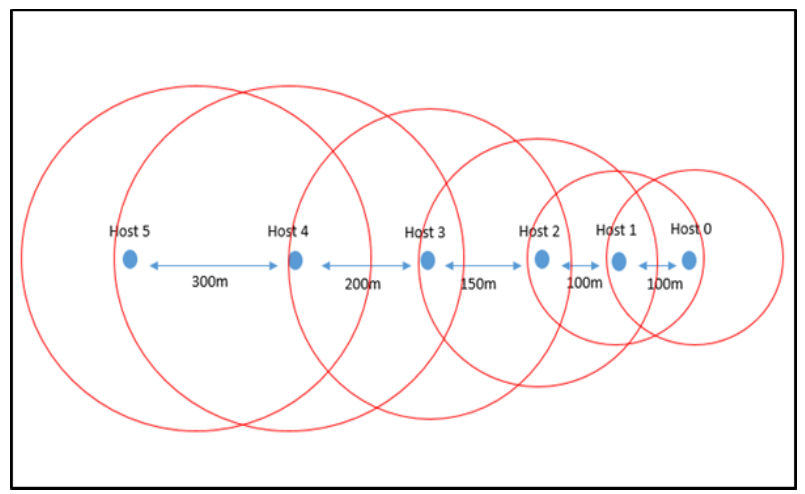

Fig.2. Dynamic Transmission Range Illustration

Figure 1 and Figure 2 shows the illustration of experiment setup for fixed transmission range and dynamic transmission range respectively. The simulation time is 500s for every experiment and all nodes are configured in static mobility. In this experiment, five sizes of packet data and packet rate are used. In Figure 1, all nodes in the simulation are configured with fixed transmission range, while in Figure 2, all the nodes are configured with dynamic transmission range. The transmission range for dynamic are assign based on the distance between the nodes. The details of the simulation are summarized in Table 2. 
Table 2: Details of Simulation Setup

\begin{tabular}{|c|c|c|}
\hline & $\begin{array}{l}\text { Fixed } \\
\text { Transmission } \\
\text { Range }\end{array}$ & $\begin{array}{l}\text { Dynamic } \\
\text { Transmission } \\
\text { Range }\end{array}$ \\
\hline $\begin{array}{l}\text { Distance } \\
\text { Between } \\
\text { Nodes }\end{array}$ & \multicolumn{2}{|c|}{$\begin{array}{l}\text { Host } 0 \text { to Host } 1=50 \mathrm{~m} \\
\text { Host } 1 \text { to Host } 2=100 \mathrm{~m} \\
\text { Host } 2 \text { to Host } 3=150 \mathrm{~m} \\
\text { Host } 3 \text { to Host } 4=200 \mathrm{~m} \\
\text { Host } 4 \text { to Host } 5=250 \mathrm{~m}\end{array}$} \\
\hline $\begin{array}{l}\text { Transmission } \\
\text { Range }\end{array}$ & $\begin{array}{c}3 \text { sets of } \\
\text { experiments: } \\
1^{\text {st }}=3 \mathrm{~mW} \\
2^{\text {nd }}=5 \mathrm{~mW} \\
3^{\text {rd }}=7 \mathrm{~mW}\end{array}$ & $\begin{array}{l}\text { Host } 0=0.3 \mathrm{~mW} \\
\text { Host } 1=0.6 \mathrm{~mW} \\
\text { Host } 2=0.9 \mathrm{~mW} \\
\text { Host } 3=1.5 \mathrm{~mW} \\
\text { Host } 4=2.2 \mathrm{~mW} \\
\text { Host } 5=2.5 \mathrm{~mW}\end{array}$ \\
\hline Packet Size & \multicolumn{2}{|c|}{$\begin{array}{c}1,2,3,4 \text { and } 5 \mathrm{~KB} \\
(\text { Fixed packet rate }=1 \text { packet } / \mathrm{sec})\end{array}$} \\
\hline Packet Rate & $\begin{array}{l}2,4,6 \text { and } 8 \\
\text { (Fixed pack }\end{array}$ & $\begin{array}{l}\text { (Packet / sec.) } \\
\text { size = } 1 \mathrm{~KB})\end{array}$ \\
\hline
\end{tabular}

\section{Evaluation Scenario}

The experiments were evaluated at different network loads. The experiments were run by increasing the size and the rate of packet at using different transmission range.

\subsection{Evaluation Metric}

The evaluation metrics which are chosen to analyse the performance are energy consumption, PDR, throughput and transmission time delay. The evaluation metrics are explained in the Table 3 .
Table 3: List of Evaluation Metric

\begin{tabular}{|l|l|}
\hline \multicolumn{1}{|c|}{ Name } & \multicolumn{1}{c|}{ Definition } \\
\hline $\begin{array}{l}\text { Energy } \\
\text { consumption }\end{array}$ & $\begin{array}{l}\text { Energy used by the node to transmit } \\
\text { the data }\end{array}$ \\
\hline Throughput & $\begin{array}{l}\text { The average rate of successful packet } \\
\text { that can be delivering to its } \\
\text { destination over a communication } \\
\text { channel per unit of time } \\
\text { Throughput }\end{array}$ \\
$=\begin{array}{l}\text { Received packet in bits } \\
\text { Packet } \\
\text { Delivery } \\
\text { Ratio (PDR) }\end{array}$ & $\begin{array}{l}\text { Simulation time } \\
\text { successfully received by the receiver } \\
\text { source }\end{array}$ \\
\hline Delay & $\begin{array}{l}\text { The arrival time needed by the packet } \\
\text { to its destination. }\end{array}$ \\
\hline Received Packets
\end{tabular}

\section{Result and Discussion}

Different types of tests are performed to evaluate and investigate the effects of using different packet sizes and different packet rates on the energy consumption of static node wireless ad-hoc network. Each experiment was repeated ten times to get the accurate results. The evaluation and analysis was made by using the average result value of all the ten experiments.

\subsection{Energy Consumption}

The result of energy consumed by the network when using different packet size are illustrated as shown in Figure 3. The higher the packet size, the lower the energy used due to the fact of high packet collision and packet corrupted. Packet corruption rate increase as packet size increase due to the packet fragmentation. Packet fragmentation is a process that split the packet into smaller pieces, so that packets can pass through a link with a maximum transmission unit (MTU) compared to the original size. For example, if we transmit packet of 2048B and 5120B in size. For 5120B the packet will be split into 4 packets compared to 2048B, it only split into 2 packets. Since the MTU size is 1500B. So for higher packet size, more packets are produce compared to smaller packet size. Therefore, the probability of packet loss or drop due to packet collision and corrupted are high for larger packet size. In turn, the energy consumption will be low. From the graph also 
shown that dynamic transmission range give better energy saving compared to fixed transmission range.

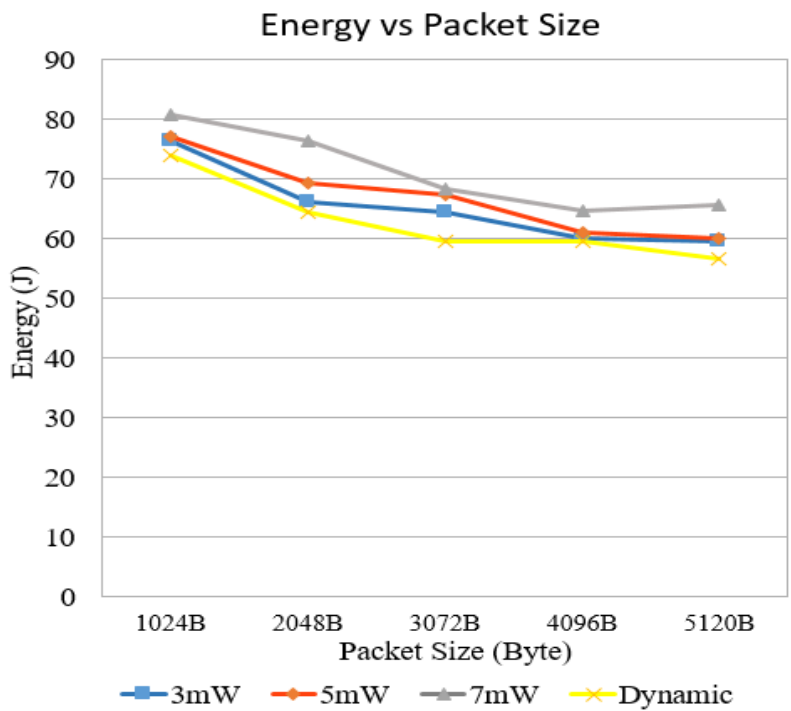

Fig. 3. Energy vs. Packet size

Figure 4 shows the graph of energy consumption over packet rate for different transmission range which is $3 \mathrm{~mW}, 5 \mathrm{~mW}, 7 \mathrm{~mW}$ and dynamic transmission range. Packet rate is the amount of packet sent per unit of time during the communication process. In a simple word, it means how many packets per second (packet/sec). The higher the packet rate, the lower the energy used due to the fact that high probability of collision happen due to fast transmission rate and the network will be congested. When collision happened, the rate of successful packet arrived at the destination will decrease, giving rise to more number of packets being loss or drop. Furthermore, increasing the packet rate make the number of packet waiting in the MAC layer buffer to be sent also increase. The entire packets that exceed their waiting time limit are discarded by the MAC layer. When the number of packet loss is high, the energy used is low. The graph also shows that the energy consumption for dynamic transmission range is better than energy consumption for static transmission range.

From the Figure 3 and Figure 4, the graph shows that the higher the packet size and the higher the packet rate, the energy consumption will be low. This is due to more packet are loss due to high traffic generated. Since we used UDP that does not provide retransmission, the energy used will be low due to less number of packets were completed the transmission task. Although it is achieve our target to get lower energy consumption, but in term of PDR and throughput, it will be low. Hence to avoid this problem happen to our purposed method that will be implemented in VANET, an optimization method will be used. The optimization method are used to find the most suitable packet size and packet rate that can give higher PDR and throughput and at the same time the energy consumption will be lower compared to the existing method.

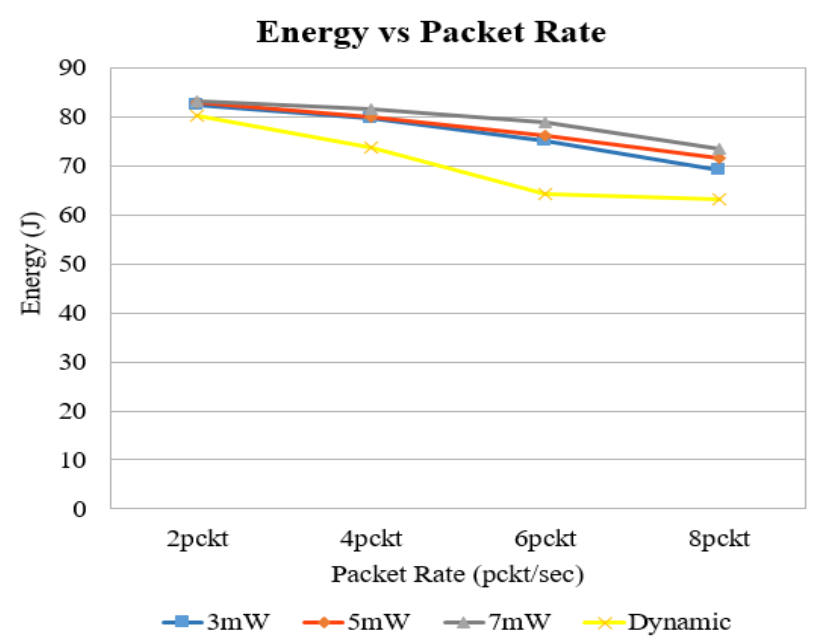

Fig. 4. Energy vs Packet Rate

\subsection{Throughput and PDR}

The result of throughput (bps) versus different number of packet size are shown in Figure 5 and the result of throughput versus different packet rate are shown in Figure 6. Both of the figure, which is Figure 5 and Figure 6 depicts that an increasing number of packet rates and packet size, increase the amount of data injected into the network. This data injection leads to an increase in throughput. The larger the packet rate, the larger the throughput and the larger the packet size, the larger the throughput.

Throughput and packet delivery ratio (PDR) are directly proportional to each other. When the PDR increase, the throughput also increase. Figure 7 demonstrate the result of PDR versus packet size and Figure 8 show the result of PDR over packet rate. It shows that, when packet size or packet rate increase, the PDR also increases.

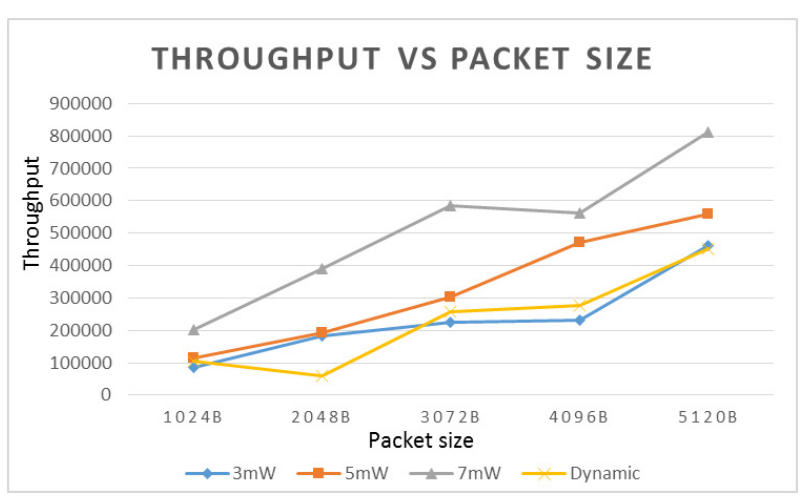

Fig.5. Throughput vs. Packet Size 


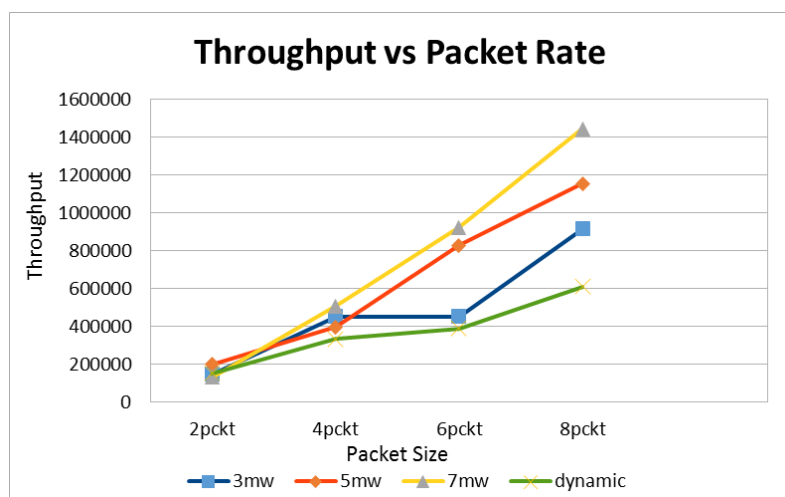

Fig.6. Throughput vs. Packet Rate

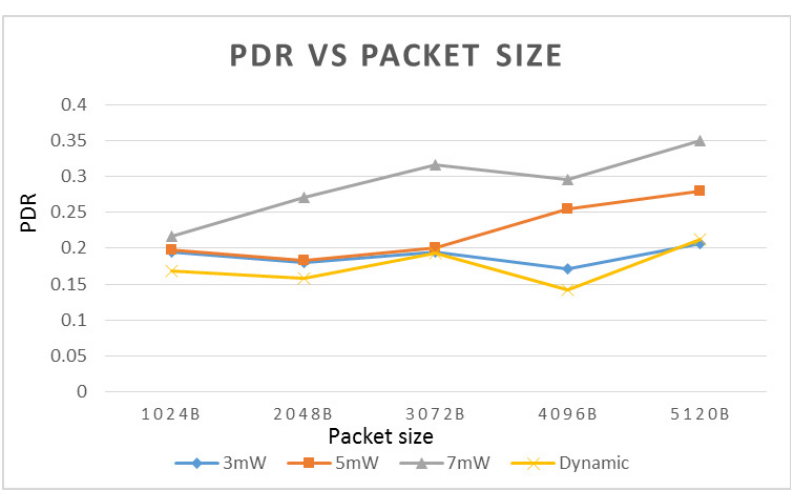

Fig.7. Packet Delivery Ratio (PDR) vs. Packet Size

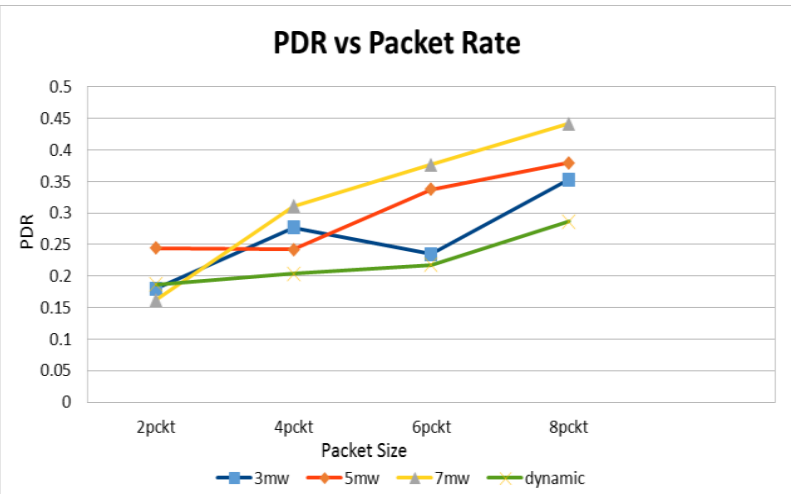

Fig.8. Packet Delivery Ratio (PDR) vs. Packet Rate

\subsection{Transmission Time Delay}

The transmission time delay for larger packet size is higher than the transmission time delay for smaller packet size as shown in Figure 9. The higher the packet size, the higher the delay. For example, delay for 5120B packet size is higher compare to $4096 \mathrm{~B}$ packet size as shown in Figure 9. This is because the transport layer of each node needs more time to transmit a larger packet size compare to smaller packet size.

Moreover, increasing the packet rate increase the number of packets waiting in the Mac layer buffer to be sent, this in turn leads to an increasing in the transmission time delay. Increasing the packet rate also make the transport layer of each node need more time to send the data. This is the reason why transmission time delay in Figure 10 increase with the increasing number of packet rate. This is true for all cases.

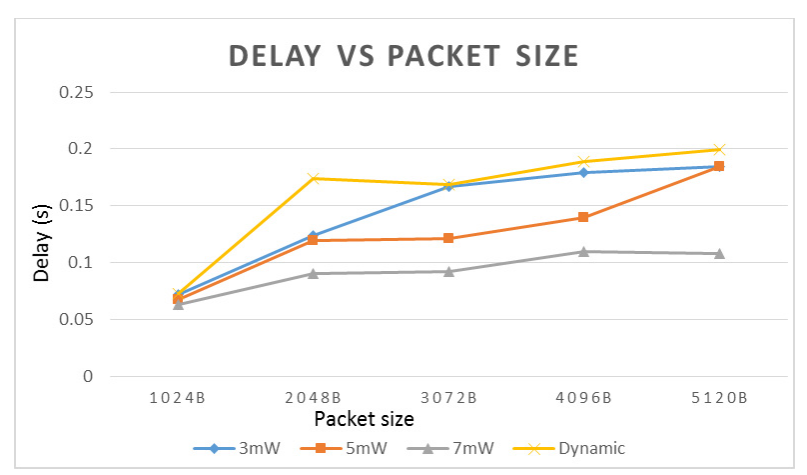

Fig.9. Transmission Time Delay vs. Packet size

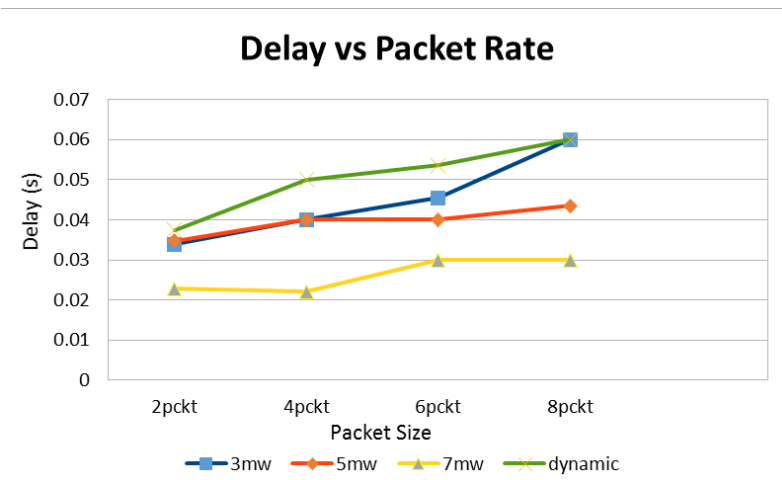

Fig.10. Transmission Time Delay vs. Packet Rate

\section{Conclusion}

This paper investigates the energy saving efficiency of dynamic transmission range and static transmission range in static mobility node wireless ad-hoc network which is prerequisite experiments before further experiment on VANET can be carried on. The simulation results prove that dynamic transmission range gives better energy consumption compared to static transmission range, so it is worth it to carry out the subsequent experiments on VANET. Although the result of PDR, throughput and delay from this experiments shows that dynamic transmission range is lower compared to static transmission range, this reasons is not a backstop to the subsequent experiments in VANET. This is due to the fact that been proved by the authors in the paper [5][6][7][8][9], which is the performance of dynamic transmission range in VANET is better compared to the performance of static transmission range. This is because, the application of VANET use a travelling cars as nodes in a network to create a mobile network. That means, the node in VANET used a high speed mobility node and the location of the nodes frequently change. This maybe the factor why the dynamic transmission range in this experiment give lower performance. In VANET the information may be 
directly sent to the destination since the nodes frequently change the location. The nodes did not need to use the intermediate node to send or receive the information. Hence less packet maybe drop or loss during the communication process compared to this experiments. This is because by using dynamic transmission range on static mobility ad-hoc network in this experiments, the transmitted data need to use intermediate node to reach the destination. The transmission range of dynamic transmission range are assign based on the distance between the nodes. Hence, more data might be dropped and lost during the transmission process since the data need to travel from one node to another node to reach the destination. This is the reason why the PDR, throughput and delay for the dynamic transmission range in this experiment give lower result compared to static transmission range. Since the objective of this experiment was to investigate and compare the energy consumption between the dynamic and fixed transmission range, therefore the result of PDR, throughput and delay of this experiment were not take into consideration.

\section{Acknowledgment}

The research reported in this paper is supported by Research Acculturation Grant Scheme (RAGS) [Grant number: 9018-00082]. The author would also like to express gratitude to the Malaysia Ministry of Higher Education (MOHE) and University Malaysia Perlis for the facilities provided.

\section{References}

1 S. S. . S, "A Survey on Wireless Ad Hoc Network Issues and Implementation," 2016 Int. Conf. Emerg. Trends Eng. Technol. Sci., pp. 1-6., 2016.
2 D. Helen and D. Arivazhagan, "Applications , Advantages and Challenges of Ad Hoc Networks," vol. 2, no. 8, pp. 453-457, 2014.

3 S. Verma and P. Singh, "Energy Efficient Routing in MANET : A Survey," vol. 3, no. 2, pp. 39713977, 2014.

4 "OMNeT++ Discrete Event Simulator - OMNeT++ Discrete Event Simulator," 2014. [Online]. Available:

https://omnetpp.org/component/jdownloads/downlo ad/32-release-older-versions/2290-omnet-4-6source-ide-tgz. [Accessed: 11-Nov-2016].

5 R. Chai, B. Yang, L. Li, X. Sun, Q. Chen, Rong Chai, Bin Yang, Lifan Li, Xiao Sun, and Qianbin Chen, "Clustering-based data transmission algorithms for VANET," 2013 Int. Conf. Wirel. Commun. Signal Process., no. 61102063, pp. 1-6, 2013.

6 R. hen, H. Yang, W. Jin, and A. Regan, "Dynamic Transmission Range in Inter-Vehicle Communication with Stop-and-Go Traffic," 2010 IEEE Intell. Veh. Symp., pp. 1-6, 2010.

7 J. M-Y. Lim, Y. C. Chang, J. Loo, and M. Y. Alias, "Improving VANET Performance with Heuristic and Adaptive Fuzzy Logic Scheme," Wirel. Pers. Commun., vol. 83, no. 3, pp. 1779-1800, 2015.

8 D. B. Rawat, D. C. Popescu, and S. Member, "Enhancing VANET Performance by Joint Adaptation of Transmission Power and Contention Window Size," vol. 22, no. 9, pp. 1528-1535, 2011.

9 M. M. Artimy, "Local Density Estimation and Dynamic Transmission-Range Assignment in Vehicular Ad Hoc Networks," IEEE Trans. Intell. Transp. Syst., vol. 8, no. 3, pp. 400-412, 2007. 